\title{
Resultados de una intervención para la re-inserción familiar de menores bajo custodia del Estado
}

\section{Ruth Vallejo Castro ${ }^{1}$ y Cinthya Berenice Rodríguez Piedra ${ }^{1}$}

\section{Resumen}

En el presente trabajo se muestran los resultados obtenidos en la ejecución de un programa de intervención creado para la re-inserción de niños, niñas y adolescentes del Sistema DIF Michoacán, en familias que hayan solicitado su re-integración. En la ejecución del programa, se implementaron actividades encaminadas a facilitar la re-inserción de los menores. Se trabajó con 15 familias, durante los encuentros mensuales de convivencia familiar con los mejores bajo custodia establecidas por el DIF estatal. La imple-

1 Universidad Michoacana de San Nicolás de Hidalgo, ruthvc4@hotmail.com 
mentación de la actividad Ilamada Diario de convivencia familiar permitió plasmar las vivencias significativas ocurridas a partir de las actividades realizadas propiciando el desarrollo de un vínculo afectivo entre ellos. Concluimos que en la medida en que previamente se prepare al núcleo familiar y al menor para conformar un nuevo sistema familiar, la re-inserción se da de mejor manera ya que el niño puede elaborar previamente el trauma que viene cargando desde su familia de origen.

Palabras clave: programa de intervención; menores de edad; re-inserción familiar; diario de convivencia familiar.

\section{Abstract}

This work shows the results obtained in the execution of an intervention program created for the reintegration of children and adolescents from the DIF Michoacán System, in families that have requested their reintegration. In the execution of the program, activities aimed at facilitating the reintegration of minors were implemented. We worked with 15 families, during the monthly meetings of family coexistence with the best in custody established by the state DIF. The implementation of the activity called Family life diary allowed to capture the significant experiences occurred during the activities carried out, promoting the development of an emotional bond between participants. We conclude that reinsertion occurs in a better way to the extent that the family 
nucleus and the minor are previously prepared to form a new family system, since the child can previously elaborate the trauma that he has been carrying from his origin family.

Keywords: intervention program; minors; family reintegration; family life diary.

Hablar de conformación familiar nos remite tradicionalmente a situar el parentesco como el elemento básico. Sin embargo, no todas las familias están atravesadas por lazos con-sanguíneos. Según cifras del INEGI (2017), el 10.8\% de estas conformaciones son un conjunto de personas que viven juntas pero que no guardan relación de parentesco directo. En este sentido, cada familia debe ser estudiada con base en sus propios procesos, considerando las estructuras que la determinan para poder entender su conformación y, en un momento dado, su desintegración.

Los factores que originan la desintegración familiar son sociales, económicos, la migración o la muerte de algún miembro de la familia, la ausencia del padre o la madre debido a algún proceso judicial o la separación de ambos, entre otros.

Dentro de los factores que conllevan a una ruptura familiar por el desequilibrio que sufren sus miembros, encontramos las adicciones, la prostitución, las infidelidades de pareja, el machismo y la violencia familiar. Esta última hace referencia al maltrato físico y/ 
o psicológico que sufren algunos miembros de la familia, incluyendo el abandono, el rechazo y la discriminación.

Los factores económicos que influyen en la desintegración familiar incluyen al desempleo, las crisis económicas y las familias en las que ambos padres trabajan, lo que provoca un distanciamiento entre ellos y con los hijos. El distanciamiento con los niños pequeños produce desequilibrios emocionales, mientras que en los adolescentes los lleva a presentar conductas erráticas.

Con base en estos factores, las estructuras familiares se conforman de diferentes maneras. Minuchin y Fishman (1981/2004), por ejemplo, nos muestran los siguientes 10 tipos de familias:

a) Familias de pas de deux. Son familias compuestas por dos personas, por lo que los individuos contraen una recíproca dependencia casi simbólica. Pueden estar conformadas por la pareja parental ante la presencia del síndrome del nido vacío o la conforman alguno de los padres con un hijo.

b) Familias de tres generaciones. En este tipo de familias se encuentran conviviendo los abuelos, padres e hijos estableciendo una relación estrecha.

c) Familia con soporte: En este tipo de familias los hijos mayores toman a su cargo las responsabilidades de sus padres. Común en 
familias muy extensas donde los padres delegaban responsabilidades por lo regular en los hijos mayores quienes se encargaban del cuidado de los hermanos menores.

d) Familia acordeón. Este tipo de familias se caracterizan por la presencia de migrantes. Uno de los progenitores se ausenta por largos periodos de tiempo tomando su lugar el cónyuge que se queda a cargo de los hijos o, en su defecto, una tercera persona que asume las funciones de cuidado y protección de los niños.

e) Familias cambiantes. Estas familias se caracterizan por una inestabilidad social por cambio de domicilio constante lo que origina que continuamente se estén adaptando a nuevas costumbres y contextos donde los niños no pueden entablar relaciones afectivas duraderas con menores de su edad.

f) Familias huéspedes. Son familias que acogen a un menor de manera temporal es decir, por un tiempo determinado. En ocasiones la familia lo incorpora al sistema familiar como si no fuera un huésped.

g) Familias con padrastros o madrastras. Son familias reconstruidas en las que los padres pueden tener hijos de un matrimonio anterior y/o adoptar los hijos de la pareja con la que reinician su vida. Esto crea distintos tipos de 
alianzas entre los integrantes de la familia que puede llegar a dificultar su convivencia.

h) Familias con un fantasma. Son familias que han sufrido la muerte de alguno de los progenitores o deserción de algún miembro. En algunas ocasiones estas familias viven a la luz de un duelo no tramitado donde no pueden asumir las tareas que realizaba el progenitor fallecido o ausente.

i) Familias descontroladas. Son familias donde alguno de sus miembros presenta síntomas de falta de control, ya sea alguno de los padres o los hijos.

j) Familias psicosomáticas. Cuando uno de los miembros está enfermo y la estructura familiar actúa de forma sobreprotectora, creando fusión o unión excesiva entre los miembros de la familia.

Helm Sierling (citado por Casas, 1992), incluye además a las familias en proceso de disolución, familias con integrantes psicóticos, familias con jóvenes delincuentes, familias con tendencia drogadicta, familias con integrantes de tendencia suicida y familias con niños con impedimentos psíquicos o físicos.

Las familias con las que se trabaja en el DIF Estatal Michoacán coinciden con algunas de las conformaciones descritas por Minuchin especialmente las familias huésped, las familias con padrastros y/o madrastras, las familias con fantasmas, familias descon- 
troladas y psicosomáticas, aunque también se observan las familias en proceso de disolución mencionadas por Sierling.

Los niños que están bajo resguardo del estado, por lo regular, provienen de familias disfuncionales, donde la falta de atención de los padres, los límites poco establecidos y en general un mal funcionamiento familiar hace que los menores sean recogidos del seno familiar con la finalidad de salvaguardar su integridad. En tanto la familia no se encuentre estable, el menor permanecerá bajo custodia del estado; para que éste pueda ser reintegrado, la familia tendrá que cumplir con una serie de condiciones, entre ellas las convivencias familiares.

Es importante mencionar que estos menores parten de historias de vida muy diversas, como violencia, abandono, abuso sexual, drogadicción, trata de menores, por mencionar solo algunas, historias que se anudan dentro de las Casas Hogar, donde permanecen en tanto no se da la re-inserción, con las situaciones de vida de sus pares, las cuales portan historias iguales o aún más difíciles. La separación de sus núcleos de crianza, que, aunque desfavorables, han sido su única realidad; el permanecer por largo tiempo en Casas Hogar los colocan en condiciones vulnerables con el peligro de convertirse en menores institucionalizados. Con esto nos referimos al estado psíquico del menor que vive bajo el resguardo de una institución y se piensa de esta manera "institucionalizado", trayendo como consecuencia un deterioro 
psíquico importante. Mientras más permanezca un menor en estado de institucionalización, mayor será el desafío para re-adaptarlo a la sociedad; de aquí la importancia de que el proceso de reintegración se cumpla de la mejor manera pensando en el bienestar psíquico del menor.

Aunque hablar de re-inserción del menor pudiera parecer solo un trámite, esta conlleva aspectos jurídicos que son importantes tomar en cuenta. Por ejemplo, un menor que fue retirado de su familia nuclear por maltrato, violencia o abuso sexual, por mencionar sólo algunas causas, no puede regresar si el agresor sigue formando parte de ella. En estos casos será algún otro familiar el que reclame la custodia del menor y solicite su reintegración.

Cuando los padres, algún familiar o tutor solicita la reintegración del menor a la familia, el Sistema DIF inicia con una serie de procedimientos que tiene que acatar el solicitante. Un ejemplo sería la solicitud formal ante la Procuraduría de Protección para Niños, Niñas y Adolescentes (PPNNA), dado que se realiza todo un trámite legal. El o la solicitante debe proporcionar además información, vía trabajo social, acerca de las condiciones del hogar donde habitará el menor con el fin de asegurar que el niño reciba los cuidados y atenciones necesarias para su sano desarrollo. Estas condiciones incluyen el espacio físico donde habitará, los servicios con los que cuenta la vivienda, el acceso a la educación que tendrá el menor, el cuidado de su 
salud y los medios económicos con los que se solventará su manutención.

Por su parte, el departamento de psicología somete a evaluación al solicitante mediante pruebas psicométricas y proyectivas, incluida la entrevista psicológica, con la idea de evaluar las condiciones psíquicas de aquel que se hará cargo directamente del menor. Una vez realizada la evaluación psicológica del solicitante y al no haber encontrado ningún signo de riesgo para el menor, se inicia un periodo de convivencias con la finalidad de promover la interacción sana y la eventual re-inserción del menor al núcleo familiar.

El espacio de convivencias permite una evaluación de la dinámica familiar, determinante para que el estado reintegre o no al menor con su familia. Con base en esto los psicólogos del DIF realizan la observación de la convivencia familiar, tomando notas que serán enviadas a los abogados para determinar si se autoriza o no la re-inserción del menor. Por otra parte, el espacio de convivencia se convierte en un espacio idóneo para que, mediante la intervención de programas psicológicos, se propicien formas de interacción positivas entre el menor y la familia de acogida.

Una de las actividades que cobró mayor importancia fue la realización de un Diario de convivencia familiar, mismo que se implementó dentro de un programa de intervención, ya que permitía plasmar las distintas acciones realizadas en el programa de convivencia quedando como un elemento testigo de la interacción pero también de resguardo de los momen- 
tos que pasaban los menores con la familia. Lo que se planteaba fue la escritura de las historias por las que transitaba la familia al momento de convivencia, un diario colectivo que rescató lo mejor de esos momentos.

Según Martín-García (1995), la historia de vida, como una modalidad del diario, es una técnica de investigación cualitativa donde el investigador transcribe y analiza el relato de una persona sobre acontecimientos y vivencias destacadas del sujeto. En este caso, es el propio sujeto, el menor, y uno o varios familiares, quienes escriben y relatan el acontecimiento de la convivencia y lo que esto le significó. Menciona el autor que, "lo que se intenta con esta técnica de historias es dibujar el perfil cotidiano de la vida de una persona o grupo de personas a lo largo del tiempo" (Martín-García, 1995, p. 42).

En este sentido, el diario trabajado entre los miembros de la familia recoge las vivencias ocurridas durante los encuentros, potencia los momentos buenos vividos y los rememora en cada encuentro, posibilitando que, con el transcurso de la convivencia, se vaya fortaleciendo un vínculo familiar que se establece inicialmente de manera poco sólida.

Si bien no existen directrices suficientemente claras con relación al diseño, aplicación y análisis de un diario, si podemos mencionar que la técnica permite la introspección a partir del tiempo y el espacio que se dan los sujetos para escribir aquello significativo para ellos. Molina (citada en Jarque, 2013) detalla entre los 
beneficios de quienes escriben un diario que: "ganan en autoconciencia, autoconocimiento y autoestima, además de incrementar la comprensión hacia sí mismos". Plasmar sobre un diario familiar las vivencias que acontecen durante su reunión permite un conocimiento de la familia en cuanto a la forma como interactúan. Además, sirve como memoria colectiva de aquello que aconteció durante los encuentros en la medida en que codifica los sentimientos y pensamientos de aquellos que se encontraron. Los diarios de convivencia y las historias de vida tienen puntos de encuentro muy semejantes, de hecho se menciona que los diarios en general son una forma de trabajar con historias de vida.

Como mencionan Puyana y Barreto (1994):

La historia de vida proporciona una lectura de lo social a través de la reconstrucción del lenguaje, en el cual se expresan los pensamientos, los deseos y el mismo inconsciente; constituye, por lo tanto, una herramienta invaluable para el conocimiento de los hechos sociales, para el análisis de los procesos de integración cultural y para el estudio de los sucesos presentes en la formación de identidades. (p. 187)

Son estos pensamientos, deseos y manifestaciones de lo inconsciente los que cobran particular relevancia al momento de la escritura y del dibujo en el diario. Los dibujos son fundamentales para poder plasmar los recuerdos dentro de un diario ya que quedan como huellas indelebles de algo que aconteció 
en cierto momento y lugar. Al momento de recuperar esos recuerdos, se significan de manera distinta. Aquel miembro de la familia que toma el diario para escribir sobre él, escribe sus pensamientos y/o sentimientos en torno a la convivencia a modo muchas veces de catarsis. Sobre esta base y concepción es que se pidió a las familias la escritura de aquello representativo durante la convivencia, un diario familiar donde pudieran registrar lo que aprendieron e hicieron durante las actividades del programa de intervención.

\section{Método}

\section{Participantes y escenario}

El programa de intervención para la re-inserción de niños, niñas y adolescentes bajo custodia del Estado a sus familias y/o tutores, se implementó en el Centro de Cultura y Artes perteneciente al DIF Estatal de Michoacán. Se trabajó en el área verde del Centro en dónde son convocadas las familias para convivir con los menores, cuyas edades oscilan entre los pocos meses de nacidos y la cercanía a la mayoría de edad.

Cada actividad del programa se trabajó con 15 familias junto con los menores durante distintos momentos de las convivencias. Para el desarrollo de las distintas actividades se contó con el apoyo de 4 estudiantes de servicio social de la carrera de Psicología de la Universidad Michoacana de San Nicolás de Hidalgo. 
Procedimiento

El objetivo general de la creación e implementación del programa fue fomentar la integración familiar y el reconocimiento de cada uno de los miembros de la familia para facilitar la re-inserción de los menores. Como objetivo específico, se buscó propiciar la comunicación, enseñanza de valores y unión familiar para potenciar una mejor dinámica familiar. Con base en este objetivo, se trabajó una vez al mes, durante alrededor de 9 meses, con cuatro familias por sesión, desarrollándose 9 sesiones de convivencia. Cada sesión duró cuatro horas de intervención, realizándose durante los meses de febrero a octubre del 2017. Se les dio seguimiento a los menores hasta que éstos fueron re-insertados en las familias.

La actividad que se detalla a continuación llamada Diario de convivencia familiar, conjunta las distintas actividades trabajadas durante la implementación del programa. Esta actividad se realizó en cada encuentro que tuvieron los menores con la familia, la cual sirvió para dar seguimiento al desarrollo de las convivencias y el efecto de las actividades que se desarrollaron durante la implementación del programa para las familias.

Esta actividad tenía una duración de aproximadamente 20 minutos. El tema a trabajar consistía en reelaborar recuerdos mediante la escritura, el dibujo y la observación de una fotografía. El objetivo general de la actividad fue que, mediante la escritura, la elaboración de dibujos conjuntos y la observación de fotos, las familias 
lograran evocar y remembrar recuerdos vividos dentro de la convivencia. Como objetivos específicos se plantearon: (a) que mediante la realización de dibujos colectivos plasmados en el diario, las familias desarrollen actividades conjuntas que posteriormente les sirva de recuerdos positivos; (b) que mediante las fotos presentadas construyan un recuerdo en común y (c) que mediante la escritura en un diario entiendan la importancia de plasmar recuerdos y acontecimientos ocurridos durante la convivencia que permitan la creación de un vínculo familiar.

El desarrollo de la actividad consistió en lo siguiente:

- Antes de finalizar el tiempo de convivencia, se le entregó a cada familia su Diario de convivencia familiar.

- Se invitó a los integrantes de cada familia a que escriban en este diario sus recuerdos y la manera como se sintieron durante las actividades. Se dejaba la libertad de que cada miembro de la familia escribiera o dibujara lo que quisiera con base en la indicación dada.

- Terminada esto, se les entregó una fotografía tomada de la última convivencia, recortada a manera de rompecabezas para que entre todos la armaran y al completar la imagen recordaran el momento que plasma la fotografía.

- Posteriormente se les pedía que pegaran la fotorompecabezas en su diario y escribieran lo que esa foto les evocaba. 
El material que se utilizó fue una libreta de raya, colores, lápices, goma y pegamento así como fotografías tomadas a la familia durante las convivencias.

\section{Resultados}

Los resultados se construyeron a partir del análisis de lo escrito en el diario familiar y de entrevistas realizadas a diferentes miembros de las familias. En su conjunto, estos resultados se pueden dimensionar en dos ámbitos: por una parte, los menores que iban a ser reintegrados y por otro las familias, padres o tutores que habían solicitado la reintegración.

Uno de los resultados es el relativo al reencuentro de hermanos que habían sido acogidos dentro del mismo Sistema DIF pero en casas hogar diferentes. Ante la llegada del día de convivencia los menores se hacían grandes expectativas respecto al encuentro por el hecho de ver a sus hermanos, pero principalmente por ver aquellos familiares que les brindaban una oportunidad de re-inserción.

Cuando la convivencia era entre padres e hijos, los encuentros producían llanto al momento de llegada, alegría cuando estaban juntos y tristeza cuando nuevamente tenían que separarse. Cabe mencionar que algunos de los menores fueron retirados de su núcleo familiar por falta de condiciones necesarias y suficientes para el bienestar del menor. Habiéndose visto forzados a separarse, los encuentros resultaban ser 
algo muy anhelado tanto por los menores como por sus padres.

En aquellas ocasiones en que los menores eran solicitados para su reintegración por familiares cercanos, los niños llegaban tímidamente, casi no hablaban ni convivían con el familiar debido a que apenas lo conocían o no lo recordaban.

Antes de la aplicación del programa los niños llegaban con su familiar, platicaban durante algún tiempo, una hora aproximadamente y comenzaban a almorzar. Después, la interacción de la familia se tornaba distante, no sabían que platicar o decir y, en algunos casos, se veía un claro aburrimiento.

A partir de la aplicación del programa de reinserción y las actividades realizadas, los menores y las familias encontraron un espacio donde podían convivir de diferente manera ya que, de acuerdo a cada tema que se trabajó durante la aplicación del programa, se generaron unas condiciones en las cuales la manera de relacionarse entre todos llevaba implícito el conocimiento de la forma de pensar y sentir de los miembros de la familia, lo que propiciaba que los lazos afectivos se fueran tejiendo.

Las actividades desarrolladas llevaban implícito que tanto las familias como los menores interactuaran de una manera en que les permitiera el conocimiento del otro. Los temas que se trabajaron en las actividades que se implementaron a partir del programa de intervención fueron en relación a los valores, la fortaleza que se puede tener en lo individual y como 
núcleo familiar, la imagen corporal como aquello que proyecta nuestro sentir como seres humanos, el respecto mutuo, formas de expresar las emociones en nosotros y las personas que nos rodean, entre otras.

A partir de que las familias y los menores realizaron estas actividades de interacción y conocimiento, comenzaron a aparecer indicios del establecimiento de un vínculo afectivo entre ellos. Al momento de introducir, como actividad eje, el Diario de convivencia familiar, tanto los familiares como los menores encontraron un espacio común donde plasmar lo aprendido en las distintas actividades.

Entre todos escribían sus recuerdos/memorias que resultaron más significativas para ellos a partir de las actividades realizadas. Por su parte, las fotografías tomadas resultaron ser una forma de mantener vivos los recuerdos de estas actividades, mismas que se les tomaban a las familias mientras realizaban una actividad. Gracias al armado que hacían de la fotorompecabezas, los recuerdos se presentaban y podrían permanecer gracias a ser pegadas en el diario familiar, diario que era entregado en cada reunión de convivencia. Las familias al momento de armar la fotorompecabezas mostraban expectación y alegría, se establecía un vínculo de cooperación y trabajo en equipo, mientras que otros rememoraban lo que la fotografía les iba mostrando. Estas actividades implícitas en el diario, permitieron el establecimiento de una comunicación común entre los miembros de la familia además del trabajo en equipo y la apertura de relación 
entre ellos. Se notaba alegría, sobre todo en los niños, ante la remembranza de actividades pasadas. Aun cuando no todos los integrantes de las familias escribían o dibujaban en los diarios, el hecho de leerlos en familia y recordar cosas que habían hecho permitía que la unión familiar se fortaleciera en cada encuentro, mejorando de esta manera la dinámica de relación de cada una de las familias.

En este sentido, la elaboración del diario familiar venía a recoger las vivencias ocurridas durante las reuniones de convivencia potenciando los momentos buenos vividos al ser rememorados en cada encuentro. Era común encontrar a distintos miembros de las familias hojeando el diario en los días anteriores que habían convivido, leían lo que habían escrito y le daban nuevas significaciones a los momentos ahí plasmados mediante fotografía, escritura o dibujo. la implementación del programa y del diario familiar permitieron que el vínculo familiar, establecido al inicio de manera poco sólida, se fuera fortaleciendo poco a poco.

Lamentablemente y por la misma dinámica de convivencias dentro del DIF, hubo familias que no pudieron continuar con el programa debido a que sus convivencias fueron canceladas por cuestiones jurídicas; en otras familias las convivencias no fueron muchas debido a que los menores fueron reintegrados en poco tiempo por lo alcanzaron a participar en pocas actividades. Sin embargo, las familias que sí pudieron participar en la mayor parte de las actividades del programa de re-inserción, incluyendo la realización de 
su Diario de convivencia familiar hasta el final, lograron materializar en esa libreta una historia de vida que se iba entretejiendo a partir de los encuentros que tenían y los relatos que servían como huella indeleble de aquello que habían vivido y los fortalecía como familia.

\section{Conclusiones}

De todas las actividades realizadas dentro de las convivencias familiares en el Sistema DIF, el Diario de convivencia familiar sirvió como práctica integradora de las distintas actividades desarrolladas, ya que en el diario se plasmaba la manera en que se habían sentido al realizar la actividad de ese día. Cada familia mostró una manera diferente de evocar y plasmar sus recuerdos en los diarios, los niños pequeños dibujaban la actividad que habían realizado y luego la compartían con la familia, los adultos escribían algo en relación a esto y entre todos comentaban lo que habían vivido, el hojear el diario y ver lo que habían hecho otros días, hacía que evocaran nuevamente aquellas actividades, mismas que se veían reforzadas con las nuevas, lo que los llevaba a crear lazos que se fortalecían en cada encuentro.

Los miembros de las familias con las que se trabajó buscaban guardar y rememorar las actividades realizadas dentro de las convivencias, sin encontrar limitaciones que les impidieran traer sus recuerdos al presente, por lo que el uso del diario permitió rememorar las actividades $\mathrm{y}$, con ellas, los afectos que se 
mostraron en la reunión anterior reforzándolos aún más.

La implementación de este programa permitió evaluar en un primer momento y en cada caso el tipo de familia que albergaría al menor $y$, sobre todo, permitió sensibilizar a los miembros de la familia para la adecuada aceptación del menor como nuevo miembro, todo esto en la búsqueda de la mejor re-inserción de los niños, niñas y adolescentes del DIF a las familias.

\section{Referencias}

Aberastury, A. y Knobel, M. (2012). La adolescencia normal. México: Ediciones Paidós.

Casas, G. (1992). Las configuraciones familiares.

Universidad de Costa Rica. Recuperado de http:// www.ts.ucr.ac.cr/binarios/docente/pd-000210.pdf

INEGI. (2017). Estadísticas a propósito del día de la familia mexicana (5 de marzo). Recuperado de http://www.inegi.org.mx/saladeprensa/aproposito/2 017/familia2017 Nal.pdf

Jarque, J. (2013, 20 de septiembre). Los beneficios de escribir tu diario. La Vanguardia. Recuperado de http://www.lavanguardia.com/estilos-de-vida/2013 0920/54389788499/los-beneficios-de-escribir-tudiario.html

Martín-García, A. V. (1995). Fundamentación teórica y uso de las historias y relatos de vida como técnicas de investigación en pedagogía social. Aula, 7, 41-60. Recuperado de 
https://revistas.usal.es/index.php/0214-3402/articl e/viewFile/3375/3396\%C3\%A7

Minuchin, S. y Fishman, C. (1981/2004). Técnicas de terapia familiar. Buenos Aires: Paidós.

Puyana,Y. y Barreto, J. (1994). La historia de vida: recurso en la investigación cualitativa. Reflexiones metodológicas. Maguaré, 10, 185-196. doi 10.15446/mag.v0n10.14265 


\section{(c) (1)(2)}

\section{Esta obra está bajo una \\ Licencia Creative Commons Atribución-NoComercial- Compartirlgual 4.0 Internacional}

Usted es libre de compartir o adaptar el material en cualquier medio o formato bajo las condiciones siguientes: (a) debe reconocer adecuadamente la autoría, proporcionar un enlace a la licencia e indicar si se han realizado cambios; (b) no puede utilizar el material para una finalidad comercial y (c) si remezcla, transforma o crea a partir del material, deberá difundir sus contribuciones bajo la misma licencia que el original.

Resumen de la licencia https://creativecommons.org/licenses/by-nc-sa/4.0/deed.es_ES

Texto completo de la licencia https://creativecommons.org/licenses/by-nc-sa/4.0/legalcode 